\title{
Visori e galline nel Web of Beings Second Livestock fra goliardia, semiologia e filosofia
}

\author{
Bruno Surace \\ Università di Torino \\ b.surace@unito.it
}

\begin{abstract}
Second Livestock è la pionieristica invenzione di Austin Stewart, della Iowa State University. Si tratta di un visore per galline, connesso a una cabina isolante, che introdurrebbe queste ultime in un mondo virtuale decisamente migliore rispetto a quello in cui sono costrette negli allevamenti intensivi. Di tale invenzione non esistono che tracce raffazzonate - immagini, testi, un video introduttivo - in un sito web dedicato, e tutto lascia pensare che si tratti di uno scherzo, o più precisamente di un esercizio artistico volto a polemizzare sulle condizioni del pollame di allevamento. In questo saggio tuttavia trattiamo Second Livestock volutamente come un prototipo realistico, indagando le specificità dell'animal-centered-design e le relative contraddittorietà di tale paradigma. Second Livestock viene analizzato a partire da ciò che del progetto ci è dato in misura testuale, e nel contempo l'analisi semiotica è affiancata da tutte le problematiche filosofiche ed etiche che ne scaturiscono. L'analisi ci può dire molte cose sullo stato attuale del dibattito sulla condizione animale, sul rapporto fra tecnologia e ideologia, sullo statuto e l'immaginario connessi ai geo-media. Il risultato è un nuovo paradigma di lettura dell'attuale società mediale, che non è più quello esclusivo del web of things, ma che ribattezziamo, alla luce delle osservazioni condotte, web of beings.
\end{abstract}

\section{Parole chiave}

Second Livestock, Virtual Reality, Semiotica dei media, Web of Beings, animal centered design

\begin{abstract}
Visors and chickens in the Web of Beings Second Livestock between goliards, semiotics and philosophy - Second Livestock is the pioneering invention of Austin Stewart, of Iowa State University. It is a viewer for hens, connected to an insulating cabin, which would introduce the latter into a decidedly better virtual world than the one in which they are forced on intensive farms. Of this invention there are only botched traces images, texts, an introductory video - in a dedicated website, and everything suggests that it is a joke, or more precisely an artistic exercise aimed at polemizing the conditions of farmed poultry. In this essay however we treat Second Livestock deliberately as a realistic prototype, investigating the specificities of animal-centered-design and the relative contradictions of this paradigm. Second Livestock is analyzed starting from what the project is given to us in textual terms, and at the same time the semiotic analysis is flanked by all the philosophical and ethical problems that arise. The analysis can tell us many things about the current state of the debate on the animal condition, the relationship between technology and ideology, the status and the imagery connected to the geo-media. The result is a new paradigm of reading of the current media society, which is no longer the exclusive one of the web of things, but which we rename, in the light of the observations made, web of beings.
\end{abstract}

Key Words

Second Livestock, Virtual Reality, Media Semiotics, Web of Beings, animal centered design 


\section{Ocula ${ }^{21}$}

Geosemiotica: dai locative media, alle immagini diffuse, ai big e small data

Bruno Surace I Visori e galline nel Web of Beings I DOI:10.12977/ocula2019-22

\section{Sommario}

1. Solo uno scherzo?

2. Il sito

3. Realtà virtuale fra antropomorfismo e antropomorfizzazione

4. New media, animali, schermi e libertà

5. Cyberpunk chicken

6. Conclusioni: dal web of things al web of beings

Bibliografia 


\section{Dcula ${ }^{21}$}

Geosemiotica: dai locative media, alle immagini diffuse, ai big e small data

Bruno Surace I Visori e galline nel Web of Beings | DOI:10.12977/ocula2019-22

\section{Solo uno scherzo?}

Nel footer del curioso sito www.secondlivestock.com è segnalato "Copyright 2012”. Si tratta di una data lontana, stando alla velocità di mutamento della web culture. Il sito da allora pare del tutto trascurato, eppure periodicamente i suoi contenuti sono balzati alle cronache di internet. ${ }^{1}$ Il progetto è peculiare: Second Livestock è il brand che sta dietro a visori - in stile Oculus Rift $^{2}$ - progettati esclusivamente per le galline. Di questi avveniristici dispositivi, tuttavia, non abbiamo che qualche immagine approssimativa, una serie di entusiastiche quanto generiche spiegazioni, e il video di una presentazione condotta dall'iniziatore del progetto, Austin Stewart del College of Design della Iowa State University, ${ }^{3}$ che introdurrebbe a un'eccitata, e mai inquadrata, platea (metonimia del mondo intero) la futuristica idea. Si tratta, sostanzialmente, e fino a prova contraria, di un ben congegnato scherzo.

Quel che di Second Livestock ci interessa qui non è tanto la sua immediata realizzabilità o l'intenzione che sta alla base della sua creazione, quanto la capacità di estendere e risemantizzare un paradigma sempre più sedimentato, quello dell'animal-centered design, più nello specifico dello Animal-Computer-Interaction-Design,${ }^{4}$ verso l'orizzonte sempre più vicino della realtà virtuale. ${ }^{5}$ Più largamente il progetto ingenera una riflessione sullo statuto attuale (e forse esistenziale) dei geo-media. La società reticolare, quella popolata, secondo un'espressione grottesca, dai millenials è infatti, al contrario di quanto propugni la comune vulgata, votata a un movimento repentino e doppio. Da un lato vi è il progressivo miglioramento delle tecnologie come dato ormai di fatto, irrefrenabile e teso verso l'infinito (pensarci da qui a cinquanta anni pertanto ci richiede un'immane sforzo immaginativo, ed è succulenta la tentazione di affermare che a un certo punto bisognerà rallentare, se non fosse per mancanza di risorse almeno per mancanza di idee). Dall'altro, questo il dato meno riconosciuto, vi è un parallelo - non diremmo conseguente, onde evitare l'accusa di determinismo - mutare di grandi movimenti ideali e/o ideologici, che proprio attraverso tali tecnologie circolano come sistemi di valori.

1 Alcuni esempi dalla sezione "Press" del sito web comprendono testate come Economist, Huffington Post, Independent.

2 L'Oculus Rift, finanziato in fase di sviluppo da una campagna di crowdfunding Kickstarter, è attualmente uno dei visori per realtà virtuale più diffusi sul mercato.

3 Il progetto Second Livestock è segnalato anche sul profilo di Austin Stewart sul sito della Iowa State University e sul suo sito ufficiale www.theaustinstewart.com, dove peraltro si evince l'intento artistico dell'autore.

4 Sul tema si tengono anche conferenze annuali, organizzate da Ilyena HirskyjDouglas, ricercatrice alla University of Central Lancashire a Preston. Sito di riferimento è <https://www.uclan.ac.uk>.

5 Per un approccio semiotico alla realtà virtuale cfr. Belanger (2009) e Barricelli et al. (2016). 


\section{Ocula ${ }^{21}$}

Geosemiotica: dai locative media, alle immagini diffuse, ai big e small data

Bruno Surace I Visori e galline nel Web of Beings | DOI:10.12977/ocula2019-22

Second Livestock ha il pregio di intercettare vigorosamente entrambi questi movimenti, proponendosi come tecnologia assolutamente innovativa e al contempo investendo di petto alcune fra le più potenti assiologie che oggi pervadono la nostra quotidianità, e cioè quelle relative ai diritti, o più generalmente alle condizioni, degli animali. ${ }^{6}$

I motivi per i quali la provocazione intrinseca di Second Livestock non sia fino a oggi stata accolta da semiotici, mediologi, sociologi e così via, rimangono parzialmente arcani (eppure è vero che nella polla indistinta del web è spesso difficile raccapezzarsi, e che molto rimane cristallizzato in zone grigie e periferiche), tuttavia risulta evidente, stanti le premesse finora mosse, quanto possa essere fruttifero riflettervi in sede semiotica e più ampiamente filosofica.

\section{Il sito}

Il sito di Second Livestock si presenta come una pagina informativa collegata a schede relative ai punti di forza del progetto. Il logo e il banner nell'header costituiscono un chiaro rimando al famoso brand Second Life, ${ }^{7}$ uno dei primi mondi virtuali di internet (sulla scia dei MMORPG, cioè Massive(ly) Multiplayer Online Role-Playing Games), online sin dal 2003. Il logo di Second Life, una sorta di crasi visiva fra un occhio e una mano, è integrato da un becco laterale capace di risemantizzarne potentemente il contenuto. Quelle che prima erano le dita di una mano ora sono una cresta, quello che prima era un occhio umano visto frontalmente diventa l'occhio del profilo di una gallina. Il lettering e le componenti plastiche in generale sono inalterati, a corroborare il gioco di assonanza verbale, da Second Life, "seconda vita", si passa a Second Livestock, "secondo bestiame", e cioè un secondo - nuovo - modo di concepire il bestiame. Ma non solo, il design del contenuto fa in modo che la risemantizzazione sia totale, eppure non ribalti il significato del messaggio di partenza, ma ne preservi l'assiologia. Il "secondo bestiame" di Second Livestock è infatti un bestiame che vive una "seconda vita" anche nel senso di Second Life, una vita parallela, ottenuta grazie alla tecnologia. Così anche il logo muta nella denotazione ma preserva la connotazione.

Subito sotto sono visibili, incolonnate, sei sezioni cliccabili (Virtual Free Range $^{\mathrm{TM}}$, Animal-Centered Design, An Agricultural Revolution, Ecologically Sound, New Distribution Model, Profound Innovation), le quali rimandano a pagine informative che in realtà non fanno che confermare l'ipotesi che il progetto sia perlopiù goliardico, sia per via delle immagini grezze e inverosimili proposte, sia per il riferimento a studi condotti e pareri di esperti dei quali non si trova traccia bibliografica. Infine, il footer presenta le classiche sezioni Contact, Site Admin, Events, Presentations, Press, Teaser. Anche in questo caso i dati a nostra disposizione confermano l'intenzione sardonica: i numeri di telefono proposti sono 888.555.5555 e 888.555.6666, l'unico evento schedulato è datato 24-28 aprile 2014, in un'anonima location nell'Iowa, la sezione Press linka tutta una serie di fonti online che hanno dato risalto al progetto

6 Nel merito cfr. Marrone (2017).

7 Per un inquadramento teorico di Second Life cfr. Gerosa (2007). 


\section{Ocula ${ }^{21}$}

Geosemiotica: dai locative media, alle immagini diffuse, ai big e small data

Bruno Surace I Visori e galline nel Web of Beings | DOI:10.12977/ocula2019-22
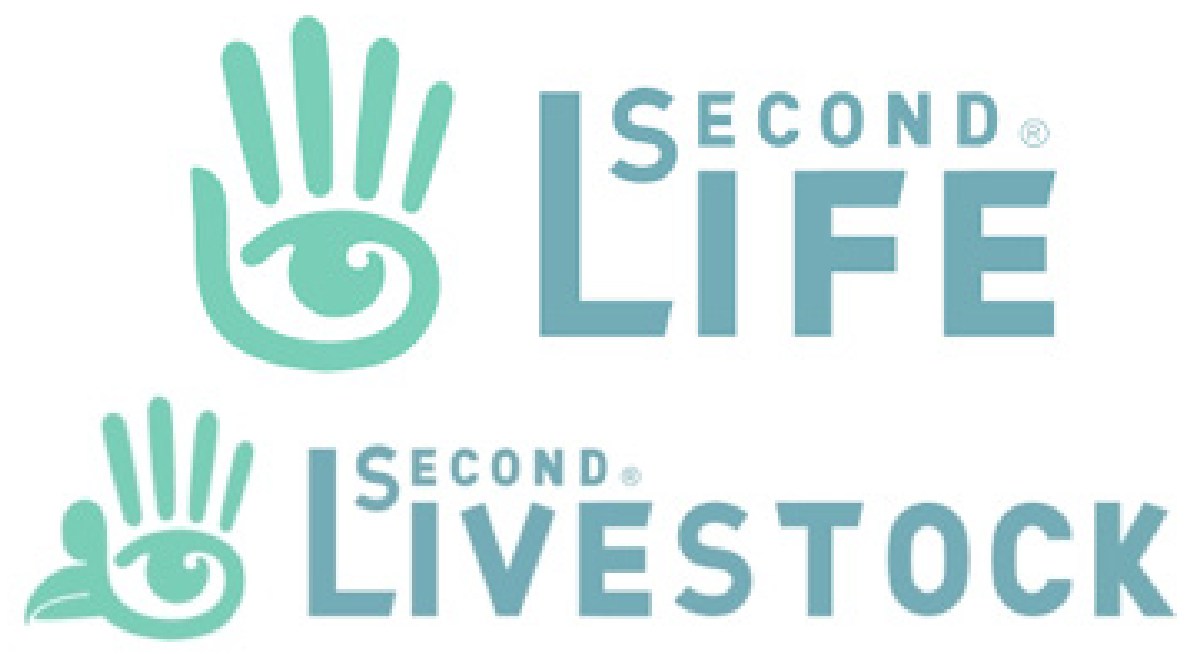

Figura 1. I loghi di Second Life e Second Livestock.

semplicemente riferendone a partire dal sito, infine il video di presentazione è montato con l'unica presenza di Austin Stewart il quale entra in un'aula universitaria accolto da un applauso chiaramente montato in post-produzione e si rivolge a un pubblico mai inquadrato (l'unico montaggio è con le slides e fra due riprese dello stesso Stewart, da destra e da sinistra) esplicando con fare quasi-messianico il progetto senza aggiungere alcun dato rispetto a quelli che si evincono dal sito.

L'asset valoriale di fondo, sul quale è costruita tutta la comunicazione, ha a che fare con la condizione del pollame d'allevamento, allevabile in "free range" virtualmente con un conseguente incremento di profitti sia in termini di felicità dell'animale, che in termini di risparmio energetico, che in termini di valore economico. La vicenda, nella sua manifesta ironia, è rilevantissima.

\section{Realtà virtuale fra antropocentrismo e antropomorfizzazione}

Fra le cose che si danno per scontate quando si parla, sempre più spesso, di realtà virtuale (da qui in avanti VR), c'è il suo essere progettata aprioristicamente per il soggetto umano: "The experience of immersive virtual reality (VR) can be considered as a communication process between human beings" (Barricelli, Gadia, Rizzi e Marini 2016: 879; corsivo aggiunto). Di forme più o meno riuscite di VR ve ne sono molte, ${ }^{8}$ ma oggi per comodità d'analisi ci concentreremo su una delle più diffuse, basata sulla presenza di un visore

8 Un esempio sono le cosiddette cave (grotte, caverne), presentate per la prima volta nel 1992. Il sistema è basato su una piccola stanza ove ogni parete, ivi compresi soffitto e pavimento, sia costituita da uno schermo con proiezioni ambientali, che teoricamente dovrebbe "avvolgere" l'utente in un mondo virtuale (con l'ausilio di suono surround). Per approfondimenti e altri esempi cfr. Taddeo (2007). 


\section{Dcula ${ }^{21}$}

Geosemiotica: dai locative media, alle immagini diffuse, ai big e small data

Bruno Surace I Visori e galline nel Web of Beings | DOI:10.12977/ocula2019-22

connesso a qualche forma di proiezione audio/video che consente una esperienza di immersività totale del soggetto coinvolto. Non c'è bisogno di scomodare il concetto di affordance o grandi trattazioni di semiotica del design per rendersi conto del sostanziale antropocentrismo che sottende questo tipo di oggetti: essi sono a misura di testa umana, e settati visivamente sulle specificità oculari della suddetta (pensati per due occhi, a una certa distanza, con un certo tipo di retina). La base dunque è un settaggio biologico e cognitivo basato su un pregiudizio antropocentrico, cioè il piazzamento dell'umano al centro dell'intero sistema. ${ }^{9}$ L'uso poi è certamente ludico, ma non solo, giacché in un contesto di VR è possibile - o sarà presto sul mercato comune - progettare oggetti e ambienti, creare opere d'arte, ed effettuare simulazioni fra le più varie (ad esempio esercitazioni di chirurgia, corsi di autodifesa, forme di fisioterapia). Il visore dunque è a tutti gli effetti un geo-medium, un po' particolare rispetto allo smartphone con le sue ormai consolidate capacità di geo-tagging. Se è vero, come sostiene Montanari, che "nella nostra cultura, ad essersi trasformata è la percezione e definizione dello stesso spazio" (2016: 162), tale percezione e definizione muta in base a due fattori. Il primo, cosa abbastanza intuitiva, è il tipo di device. Se lo smartphone dà se stesso, e quindi l'utente di cui è mcluhaniamente protesi o estensione, a una determinata geografia, il visore in VR dà una geografia all'utente. Il secondo, cosa meno intuitiva, è il tipo di utente, e con tipo intendiamo qui anche la specie a cui appartiene.

Ecco dunque che Second Livestock sposta la prospettiva, proponendo invece un simpatico visore adatto allo specifico di polli e galline; dona una geografia customizzata al pollame. L'apparecchio, stante le pochissime (e quasi sicuramente false) fonti visive che abbiamo a disposizione, si presenta come una sorta di doppio cono, una specie di clessidra che si adatta perfettamente agli occhi laterali della gallina, con un microfono che si diparte dalla struttura per andare a posizionarsi davanti al becco. Infine, ed è fondamentale a tutto l'apparato, un tapis roulant omni-direzionale darebbe la possibilità all'animale di muoversi in tutte le direzioni, correre, razzolare, starnazzare, in condizioni atmosferiche suggeritegli dalla vitrea cabina in cui è inserito. Il pollo può così sentirsi immerso in un mondo costruito a sua misura (ecco la geografia che si dà all'utente, e non viceversa) e anche, volendo, "comunicare" (questa parola utilizza durante la sua presentazione Austin Stewart) con i suoi simili connessi. Così come dimostrano sempre di più etologia e zoosemiotica infatti gli animali non solo comunicano, ma addirittura stabiliscono qualcosa che potremmo chiamare cultura sulla base delle loro comunicazioni. ${ }^{10}$

Il risultato teoretico che deriviamo dunque da Second Livestock è un cedimento dell'antropocentrismo, ma anche un rinvigorirsi dell'antropomorfismo, e cioè il perpetrarsi della logica dominante che umanizza gli animali in forza di una qualche presunta forma di rispetto. Sul piano discorsivo insomma l'operazione non è poi molto dissimile dal mettere il cappottino al proprio bar-

9 Il progetto di uno studio semiotico dell'antropocentrismo è attualmente perseguito, con risultati rilevanti, da Martinelli (cfr. ad esempio 2010, 302-326).

10 La bibliografia nel merito è sterminata. Si veda ad esempio Bonner (1980). Per uno studio recente in ottica semiotica cfr. Maran, Martinelli e Turovski (2011). 


\section{Ocula ${ }^{21}$}

Geosemiotica: dai locative media, alle immagini diffuse, ai big e small data

Bruno Surace I Visori e galline nel Web of Beings | DOI:10.12977/ocula2019-22

boncino, dal portare alle terme il proprio gatto, o dal propinare pedissequamente il mantra del "gli manca solo la parola" riferito all'animale domestico di turno. Nondimeno va rilevato come le pratiche citate, che pullulano sempre grazie a un certo intercettarsi di tecnologie e ideologie (in altre parole: i social media), si discostino da Second Livestock proprio perché quest'ultimo in qualche modo non si limita a perpetuare l'antropomorfismo, ma soprattutto lo fa in ragione di un preciso statuto etico.

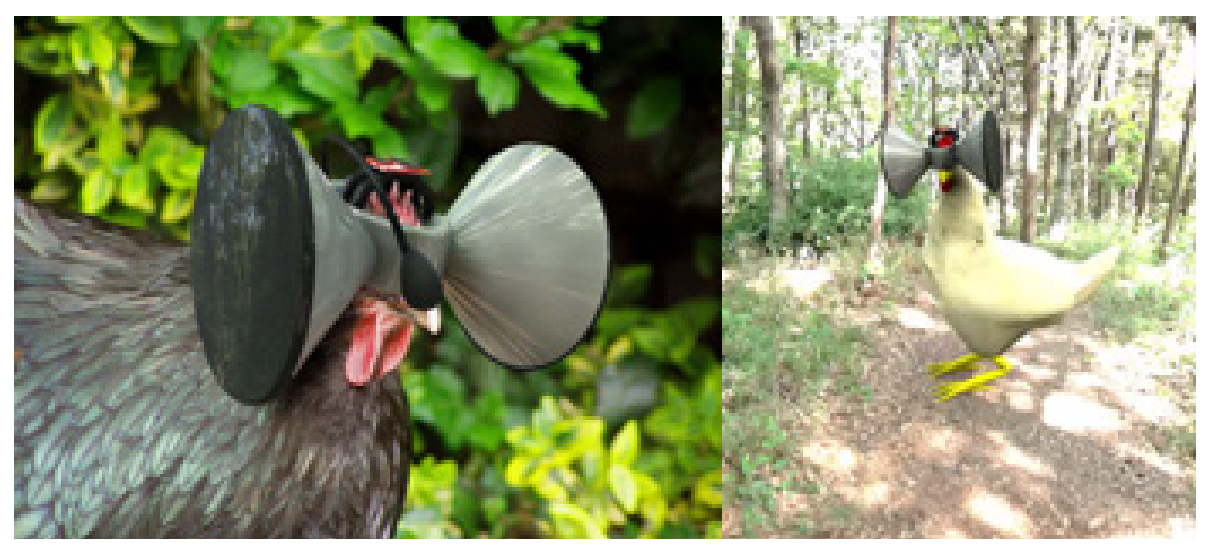

Figura 2. Il visore Second Livestock in alcune delle poche immagini disponibili. ${ }^{11}$

La condizione del pollame di allevamento è infatti tendenzialmente esiziale. Sebbene usualmente e mediaticamente si preferisca concentrarsi sul trattamento di bovini, suini od ovini, il trattamento riservato alle galline negli allevamenti intensivi non ha nulla da invidiare in termini di crudeltà perpetrata. Anche in paesi attenti alle proprie carni, come l'Irlanda ove mucche e pecore circolano libere in grandi distese erbose, la carne di pollo è prodotta in regimi crudeli ove migliaia di galline sono ammassate senza possibilità di movimento, vessate dalle infezioni derivanti da igiene scarsa o nulla, nutrite a forza e spesso imbottite di antibiotici. Second Livestock è la proposta di un cambio di prospettiva in maniera tale da abbracciare un orizzonte etico, che necessita di essere esplorata: la gallina sarebbe dotata di una personale cabina e lì la sua vita reale sarebbe sostituita giorno e notte con una vita virtuale, dove essa possa percepirsi libera, ed essere felice fino alla macellazione. Il problema bio- e semio- etico è se la gallina tuttavia sia da considerarsi libera, quando costretta in realtà nella sua sorta di vasca di vetro, cervello di memoria putnamiana, ${ }^{12}$ criogenia à la Matrix (Larry e Andy Wachowski 1999), o come dir si voglia. Su tale problema si è lungamente riflettuto in sede autenticamente umanistica, ${ }^{13}$ eppure la sua estensione al dominio animale ci pare del tutto inesplorata.

11 Il copyright di queste immagini come delle altre inserite nel saggio (ad eccezione del logo di Second Life) dovrebbe essere lo stesso del sito <https://www. secondlivestock.com>. Sono adoperate in questa sede a solo scopo illustrativo e scientifico.

12 Cfr. Putnam (1981).

13 Impossibile qui stilare una bibliografia esaustiva, essendo il tema della "definizione del reale" centrale in tutta la filosofia occidentale da secoli ad oggi. 


\section{Ocula ${ }^{21}$}

Geosemiotica: dai locative media, alle immagini diffuse, ai big e small data

Bruno Surace | Visori e galline nel Web of Beings | DOI:10.12977/ocula2019-22

\section{New media, animali, schermi e libertà}

La semiosi scaturente da Second Livestock non è dunque del tutto inedita, e si riallaccia a una precisa voga antropomorfizzante, che è poi la stessa alla base di dispositivi come la webcam per animali domestici utile ai suddetti per vedere il padrone quando è fuori casa. La presunzione di fondo è che certi meccanismi percettivi e propriocettivi, e con essi un'intera timia, tutte condizioni fondamentali nell'Interaction e User Experience Design alla base dei dispositivi digitali, ancor più se wearable, siano condivisi fra animali e umani (o, per utilizzare una terminologia in adozione, fra animali non umani e animali umani ${ }^{14}$ ). Tale assunto del senso comune si fonda e sedimenta proprio con lo spopolare nei social media di una certa retorica che interpola l'apparato sentimentale umano e quello animale a partire da testi come quello, unus pro omnibus, del leone che vede il suo "padrone" dopo molti anni e sembra abbracciarlo. ${ }^{15}$ Tutta la semantizzazione dei vari video relativi a questo episodio è tesa a dimostrare che di fatto lo "abbracci", quantunque tale atto e l'intera carica valoriale e patemica che comporterebbe sia de facto invenzione umana, pur con qualche incidenza naturale (è indubbio che certi mammiferi siano istintivamente votati al contatto con altri mammiferi). Ciò che qui più ci interessa è però indagare sulla grande contraddittorietà insita in Second Livestock, che da un lato si pone eticamente come dispositivo di forte rilevanza, e dall'altro presume che il rapporto fra una gallina e uno schermo o una schermatura ottenuti per isolamento sensoriale, possa emulare quello umano. ${ }^{16} \mathrm{~L}$ 'esperienza comune dimostra come fra animali e schermi sussista un rapporto percettivo-semiotico del tutto alterato rispetto a quello umano. Molti animali sembrano ignorare totalmente gli schermi e le rappresentazioni che li abitano, alcuni altri possono confondere gli schermi come "finestre sul mondo" (come il gattino che con la zampa cerca di prendere il pesce nel televisore), ma è caso più raro. Stesso dicasi per la musica e i rumori: definire il modo in cui un animale percepisce la musica è operazione assai complessa, se non sul piano biologico certamente su quello cognitivo (ciò vale in parte anche per l'uomo, sulla cui semiotizzazione del musicale aleggia un velo misterioso). La domanda se i cani o i gatti riconoscano i propri padroni su uno smartphone o uno schermo televisivo è diventata oggetto d'analisi recentemente, proprio con il diffondersi massivo delle tecnologie mobili in grado di replicare immagini in maniera accurata. Nicholas Dodman, della Tufts University e di DogTV - una televi-

14 Cfr. Pocar (1998).

15 Una delle tante versioni online qui:

<https://www.youtube.com/watch?v=cu_7jaJV2DE>.

16 Non ci addentreremo qui sulla questione relativa alla giustezza o meno della nutrizione a base animale da parte degli umani, ma tratteremo il terreno culturale che vi soggiace per quello che è oggi: un luogo fatto di scelte che, allo stato attuale, si basa sulla convivenza di persone che mangiano carne e prodotti alimentari a base animale e persone che scelgono di non farlo. È indubbio, comunque, che uno degli obiettivi di Second Livestock sia proprio quello di far riflettere su questa convivenza "scomoda" e sempre più tesa. 


\section{Ocula ${ }^{21}$}

Geosemiotica: dai locative media, alle immagini diffuse, ai big e small data

Bruno Surace I Visori e galline nel Web of Beings | DOI:10.12977/ocula2019-22

sione disegnata per i cani, nonostante pare che questi abbiano una soglia di attenzione minima e preferiscano spendere buona parte del loro tempo guardando nel nulla ${ }^{17}$ - sostiene che alle volte i cani vedendo immagini dei propri padroni sullo schermo televisivo possano aggirare l'oggetto per cercarli. Tale percezione potrebbe essere dovuta alla grandezza dello schermo, e pertanto potrebbe non verificarsi con schermi più piccoli come quelli di tablet e smartphone. Ron Levi, sempre da DogTV, sostiene che è una questione del tutto soggettiva, e che la rispondenza dipende dal cane, il quale è strutturalmente improntato su una riconoscibilità del mondo del tutto differente rispetto a quella umana. È ad esempio ormai dimostrato come nel naso di un cane risiedano oltre 220 milioni di recettori olfattivi, al contrario dei 5 milioni umani. ${ }^{18}$ Semplicemente (per modo di dire): l'uomo è un animale oculocentrico, il cane no. Dietro tale differenza risiede una quantità di ineffabile, uno scarto linguistico o addirittura pre-linguistico, di cui la società dei new media - anche dei geo-media, ad oggi a preponderanza visiva, nonostante alcune deviazioni da questo percorso - sembra dimenticarsi, e che nondimeno molto difficilmente si può convogliare in un oggetto, specie se questo è di derivazione umana sul lato design.

Emergono così due componenti forti: da un lato una struttura percettiva diversa (pensiamo ancora alla visione dicromatica dei cani rispetto a quella tricromatica degli umani), e quindi una sensibilmente diversa strutturazione dei tipi cognitivi; dall'altro una dose di soggettività. Se questo vale per i cani, i quali come sappiamo si suddividono poi in centinaia di razze con diverse specificità psico-fisiche, allora vale anche per ogni altra forma animale sul pianeta, ivi incluse le galline. Ne consegue l'immane problematica soggiacente a Second Livestock che adatta un dispositivo human-centered ai polli quando invece dovrebbe costruirsi su un cambio di paradigma. L'idea che l'animal-centered design derivi da quello human-centered non è altro che, infatti, l'ennesima conseguenza di un'imperante antropomorfismo, che intacca, almeno in termini di immaginario, anche l'orizzonte dei geo-media.

\section{Cyberpunk chicken}

$\mathrm{Al}$ di là dell'ineludibile problema sul lato della progettazione, è necessario soffermarsi sul tessuto etico soggiacente a Second Livestock, per poi capire cosa possa dirci di più sull'assetto mediale contemporaneo e la sua ricezione. Robert May in The Fragile Environment riferisce di uno studio della Oxford University dedicato alla "motivazione delle galline" nel deporre uova:

In Gran Bretagna, oltre il 90\% delle galline che producono uova sono tenute in gabbie (non mi sto riferendo alle galline allevate per poi essere mangiate: queste non sono tenute in gabbia). Le galline stanno su pavimenti fatti di reti metalliche e non hanno la possibilità di appollaiarsi, di razzolare o rotolare nella polvere. Non hanno nidi dove

$17<$ http://theconversation.com/heres-what-dogs-see-when-they-watchtelevision-65000 $>$.

18 <http://news.nationalgeographic.com/2015/o5/150530-dogs-animalspsychology-technology-science/>. 


\section{Ocula ${ }^{21}$}

Geosemiotica: dai locative media, alle immagini diffuse, ai big e small data

Bruno Surace I Visori e galline nel Web of Beings | DOI:10.12977/ocula2019-22

posare le loro uova e i pavimenti metallici sono in pendenza in modo che non appena le galline lasciano cadere le uova essere rotolano in avanti e possono essere raccolte automaticamente.

Potremmo chiederci se le galline siano fortemente motivate a svolgere attività quali il razzolare e rotolare nella polvere, attività che esse certamente non possono eseguire in queste gabbie. La risposta è che esse certamente non moriranno in conseguenza di ciò e quindi chiaramente la mancanza di polvere su cui rotolare non rientra nella stessa categoria estrema della mancanza di cibo o acqua. Tuttavia, come ho affermato sopra, sebbene non sembri esserci alcuna minaccia alla salute degli animali, è possibile che essi soffrano nel senso di essere fortemente motivati. Certamente alcuni degli esperimenti sembrano evidenziare che esse sono in verità fortemente motivate a razzolare nella polvere.

Ad esempio alcuni esperimenti realizzati in Danimarca hanno dimostrato che se le galline sono tenute su pavimenti metallici senza l'opportunità di razzolare nella sabbia vera o nella segatura, esse cominciano a compiere l'azione a vuoto, sui nudi pavimenti di rete. Esse eseguono tutti i movimenti associati al razzolamento anche se non vi è alcuna traccia di polvere. [...] In Danimarca è stato dimostrato che uccelli privati dell'opportunità di razzolare nella sabbia o segatura [...] si sfogano in modo eccessivo non appena gli viene data la possibilità di rotolarsi nella segatura. [...] Un terzo tipo di esperimento è stato quello di dare alle galline la possibilità di scegliere tra gabbie con diversi tipi di pavimenti. Le galline mostrano una netta preferenza per un pavimento ricoperto di segatura rispetto ad uno di filo metallico. Se gli viene data la possibilità di scegliere tra due gabbie della stessa grandezza, le galline scelgono quella con segatura sul pavimento. Esse scelgono quest'ultima persino se la gabbia con pavimento metallico è quattro volte più grande. Anche se la gabbia con segatura è talmente piccola che non possono neanche voltarsi una volta entrate, esse continuano a preferirla ad una gabbia con pavimento metallico anche otto volte più grande. Né è rilevante ciò a cui le galline sono state abituate durante l'allevamento. Galline allevate per tutta la vita su pavimenti metallici hanno esattamente la stessa forte preferenza per lo strame delle galline allevate sulla segatura. (May 1991: 72-73)

Questo lungo stralcio sprigiona un'immensa ricchezza in termini semioetici, utile all'analisi che stiamo conducendo. Esso si riferisce alle galline allevate per uova, sardonicamente sancendo che il loro trattamento sia già di per sé ben migliore rispetto a quello destinato alle galline da carne. Tuttavia possiamo presumere che le galline, non sapendo a quale programma narrativo sottostiano (se siano cioè oggetti di valore in quanto tali o se tali siano le loro uova), si comportino tutte in maniera simile. Esse prediligono, fortemente, un certo tipo di ambiente in cui vivere, e la mancanza di questo influisce sulla loro qualità esistenziale, su quella che May chiama "motivazione", $\mathrm{e}$ che potremmo addirittura arrivare a definire "felicità". Dire delle galline che esse hanno una motivazione cangiante significa definire, secondo la bipartizione proposta da Alberto Maria Cirese (1991), che esse non vivono solo una condizione di fabrilità, cioè di mera sussistenza fisiologica, ma anche una di segnicità, cioè di semiotizzazione della loro esperienza. L'orizzonte etico è di fatto riconducibile a questo punto, nel riconoscere all'animale una condizione di segnicità. Second Livestock si basa su tale assunto e, pertanto, vedendo 


\section{Ocula ${ }^{21}$}

Geosemiotica: dai locative media, alle immagini diffuse, ai big e small data

Bruno Surace I Visori e galline nel Web of Beings | DOI:10.12977/ocula2019-22

non riconosciuti i diritti associati alla segnicità, riformula i bisogni ambientali della gallina in termini virtuali. I problemi che sussistono tuttavia sono di due ordini. Il primo esula dai nostri obiettivi, e riguarda il fatto che, volente o nolente, la gallina continuerebbe a versare in condizioni di sfruttamento. Interrogarsi su tale questione significherebbe trasformare questo saggio in una riflessione di altro ordine (l'ordine primo e ultimo, per intenderci). Il secondo invece è più stimolante: Second Livestock perpetrerebbe un "inganno" nei confronti della gallina?

Il problema non è da poco, giacché rivela una sensibile differenza fra i visori umani e il prototipo per polli. Quelli umani sono concepiti come apparecchi temporanei, che consentono uno switch reversibile da e verso la realtà virtuale. "Realtà reale"19 e realtà virtuale funzionano dunque a intermittenza, si può passare dall'una all'altra, e la scelta è affidata all'utente. Tale forma di controllo, va ricordato, è subordinata al poter controllare il geo-medium. L'immaginario in stile eXistenZ (David Cronenberg 1999) per cui realtà e "finzione" si mescolino a tal punto da diventare inestricabili è quindi per ora lontano. Al contrario Second Livestock accavalla le due realtà, e fa sì che la seconda prevarichi la prima. La gallina, una volta "dentro", dentro rimarrà, fino alla sua

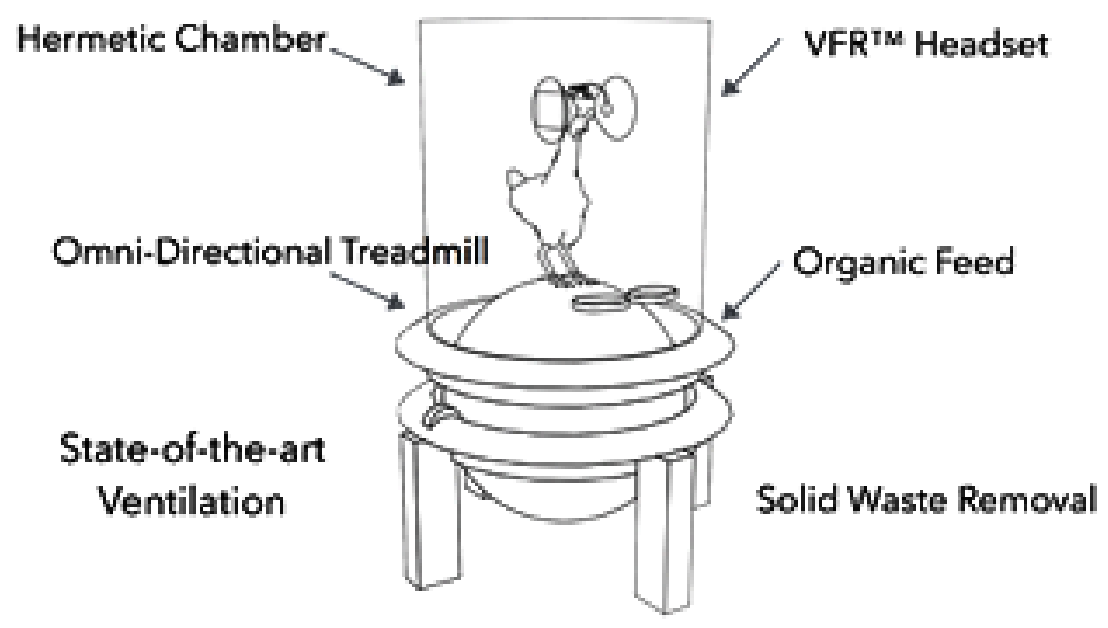

Figura 3. Una rappresentazione della cabina di Second Livestock.

fine (almeno così interpretiamo il criptico progetto).

Tuttavia, cos'è una realtà virtuale? Ci pare che il miglior modo per descriverla sia in termini semiotici. Una realtà virtuale è un ambiente interattivo costruito attraverso la giustapposizione di segni pensati ad hoc per l'utenza, al fine di immergere quest'ultima in un'esperienza in un Mondo finale $\left(\mathrm{M}_{\mathrm{f}}\right)$ che

19 Il nostro discorso oppone reale e virtuale per comodità di analisi. Siamo consci di come tale dicotomia risulti oggi superata in favore di categorie più complesse. Il laborioso dibattito nel merito non viene inserito in questa sede poiché appesantirebbe la tesi senza apportare contributi sostanziali alla nostra fattispecie. 


\section{Ocula ${ }^{21}$}

Geosemiotica: dai locative media, alle immagini diffuse, ai big e small data

Bruno Surace | Visori e galline nel Web of Beings | DOI:10.12977/ocula2019-22

sia in grado, creando un'estesia customizzata, di sostituire il Mondo iniziale $\left(\mathrm{M}_{\mathrm{i}}\right)$ in termini di sensorialità e narratività. Se quindi si tratta di un videogioco horror l'esperienza sarà disagevole e terrificante, tutto giocandosi in termini di prise esthétique (Greimas 1987), o esthésique, mentre se si tratta di un viaggio virtuale ai Caraibi o di un film porno interattivo, ${ }^{20}$ il risultato allora potrebbe essere assai gradevole. Invero se si domandasse a un operaio se preferirebbe continuare a consumarsi le ossa in attesa di una lontana pensione oppure immergersi, coi propri famigliari, in una realtà caraibica per sempre, egli potrebbe certamente discettare su questioni relative alla libertà e al suo statuto ontologico, ma potrebbe anche non considerare la proposta come un velo di Maya, e spendere nel suo mondo condiviso il tempo che gli resta (Cypher che preferisce la gustosa bistecca, a proposito, pur sapendo che è finta, perché la "realtà vera" è comunque peggio). La gallina, dal canto suo, non può scegliere con la parola, e tuttavia gli esperimenti dimostrano che posta in condizioni di scelta ambientale preferisce infilarsi in spazi minuti ma adatti a lei anziché in luoghi che non le si confanno. Da questo punto di vista Second Livestock è una soluzione da tenere in considerazione, che garantirebbe alle galline una vita felice, come esplicitato più volte nel sito, condizione che i produttori dichiarano di voler convertire in valore, e quindi in profitto, all'atto di vendita. Queste le parole del sito: "Animals do not experience the world quite like humans and what may be important features for a human in a virtual environment may be inconsequential or perhaps even threatening for another species. We have consulted with experts [Ovviamente non vi è alcuna fonte segnalata] who understand what the optimal virtual environment would look like for each animal and have tailored our environments to allow our birds to have a fulfilling existence".

\section{Conclusioni: dal web of things al web of beings}

La dissertazione fin qui condotta su Second Livestock ci dà conferma del suo essere sostanzialmente un'operazione provocatoria, che inquadriamo nel regime di vera e propria installazione, se non performance, artistica e digitale, resa possibile dai nuovi modelli di circolazione del senso nei new media. Si potrebbe addirittura parlare di un'evoluzione della net $a r t,{ }^{21}$ che abbandona una certa autoreferenzialità, il suo riferirsi a internet da internet, il suo costante - sia formale che contenutistico - rimando a una natività digitale, per darsi al mondo del web of things, se non addirittura alla nuova frontiera odierna, che ci permettiamo di battezzare come web of beings. Il nostro aver preso sul serio sin qui il progetto Second Livestock è stato dunque non solo l'adempimento alle intentiones primarie del testo - mirate a ingenerare effetti di sbigottimento, incredulità, e in seguito riflessione critica - ma anche il tentativo di dimostrare empiricamente alcune ipotesi. In prima istanza che il bacino di senso potenzialmente inespresso soggiacente a un contenuto online si può rivelare, al di là della vulgata comune, fecondo e rilevante dal punto di vista

20 Cfr. Lino (2015).

21 Cfr. Deseriis e Marano (2003). 


\section{Ocula ${ }^{21}$}

Geosemiotica: dai locative media, alle immagini diffuse, ai big e small data

Bruno Surace I Visori e galline nel Web of Beings | DOI:10.12977/ocula2019-22

teoretico. ${ }^{22}$ Ciò proprio perché tecnologia e ideologia sono manifestazioni, o meta- o sovra- strutture semiosiche intimamente interrelate, che viaggiano di pari passo in una compenetrazione continua (non una determinando l'altra, ma determinandosi l'un l'altra).

Parallelamente che la spreadability dei new media declina una nuova modalità della performance artistica, basata sulla potenzialità di circolazione del suo contenuto sia attraverso canali non ufficiali (comunità online, forum, etc), sia attraverso canali "ufficiali" (testate anche autorevoli, come l'Independent, hanno considerato Second Livestock "notiziabile"). Ciò, ovviamente, conferma il totale riassetto dell'identità delle testate giornalistiche classiche nel contesto della cosiddetta "trasformazione digitale" (Cardile, Mayer e Moder 2017).

E ancora che esiste un nesso potente, di cui la semiotica è da sempre consapevole e che tuttavia spesso viene ancora oggi ricusato, fra immaginari finzionali e costruzione sociosemiotica di dinamiche di senso nella realtà. La tendenza odierna a immaginare un futuro - e un presente - al limite dell'apocalittico è vigorosamente intrecciata con una certa sovrapproduzione di testi distopici; la lettura di Second Livestock come di una "Matrix per polli" rivela il funzionamento della installazione artistica, che mira proprio a cortocircuitare il lettore ponendolo in una situazione di multipla discrasia. Costui si trova a dover interpretare un testo che ha a che fare con finzione e realtà, ed è quindi già sottoposto a un considerevole sforzo ermeneutico, e per di più è incapace di distinguere se lo stesso testo, che si propone echianamente come narrativa naturale (1994), sia da inserirsi nel dominio di finzione o di realtà. In altre parole Second Livestock potrebbe essere un testo di finzione che racconta comunque scenari verosimili (così lo abbiamo letto noi), ma anche testo veritiero che racconta però scenari inverosimili (poiché magari irrealizzabili), o ancora un testo di finzione che racconta scenari inverosimili (da leggersi dunque come pura goliardia), o ancora un testo vero che racconta scenari verosimili (da intendersi come progetto universitario che attecchirà realmente). Districarsi fra queste possibilità inferenziali è assai complesso, ed è proprio nella mancanza di indizi rilevanti nel testo, nel suo essere necessariamente interconnesso con l'extradiegesi, causticamente deittico, che risiede lo statuto di performance artistica, non fine a se stessa.

Dall'altro lato si è rilevato come il viaggiare di pari passo di tecnologia e ideologia sia oggetto delicato, che sostanzia l'importanza della riflessione umanistica, e che con l'avvento dei wearable media e delle VR reifica una nuova "sfida per le competenze semiotiche" (Ferraro 2016: 5-13). Il paradigma nascente dell'Animal-Centered-Design rischia di rivelarsi spesso fallimentare se ancorato strutturalmente alle dominanti culturali diffuse pervasivamente proprio con la diffusione stessa dei social media. Il topos del "gattino" è, ${ }^{23}$ infatti, secondo una iniziale archeologia dei media, certamente appannaggio di fumetti e cartoni animati Walt Disney, ma si consolida e muta profonda-

$22 \mathrm{Al}$ tema è dedicato il numero di Lexia sulla viralità, curato da Gabriele Marino e Mattia Thibault.

23 Cfr. Thibault e Marino (2018) sui gattini teneri e Surace (2018a) sui gattini inquietanti. Cfr. Surace (2017 e 2018b) sulla "cucciolosità" come elemento retorico di costruzione del consenso. 


\section{Dcula ${ }^{21}$}

Geosemiotica: dai locative media, alle immagini diffuse, ai big e small data

Bruno Surace I Visori e galline nel Web of Beings I DOI:10.12977/ocula2019-22

mente a partire dal "primo" Youtube e in seguito con la diffusione dei social networks. Il modo in cui i nostri "apparati etici" sono costruiti è dunque riflesso di una certa medialità e delle nostre maniere di approcciarvisi. Riflettere di geo-media oggi significa dover fare i conti con tutte le istanze qui riassunte, che sostanziano il sistema che abbiamo definito web of beings.

\section{Bibliografia}

Barricelli, B.R. et al.

2016 "Semiotics of Virtual Reality as a Communication Process", in Behaviour \& Information Technology, Vol. 35, 2016(11), 879-896.

Belanger, Wayne D.

2009 A Semiotic Analysis of Virtual Reality, Dissertazione Dottorale, Newport, Salve Regina University.

Bonner, John T.

1980 The Evolution of Culture in Animals, Princeton, Princeton University Press.

Cardile, Dario; Mayer, Giuseppe; Moder, Pepe

2017 Trasformazione digitale. Strategie e strumenti per le PMI del futuro, Milano, Egea.

Cirese, Alberto Maria

1991 Segnicità, fabrilità, procreazione: appunti etnoantropologici, Roma, CISU.

Deseriis, Marco; Marano, Giuseppe

2003 NET.art. L'arte della connessione, Milano, Shake edizioni.

Eco, Umberto

1994 Sei passeggiate nei boschi narrativi, Milano, Bompiani.

Ferraro, Guido

2016 "Dal web al mobile: nuove sfide per le competenze semiotiche", in Guido Ferraro e Anna Maria Lorusso (a cura di), Nuove Forme d'interazione: dal web al mobile, Tricase (LE), Libellula, 5-13.

Gerosa, Mario

2007 Second Life, Roma, Meltemi.

Lino, Mirko

2015 "Porno nel virtuale. Wearable Media \& Immersive Porn”, <www.doppiozero. com>.

Maran, Timo; Martinelli, Dario; Turovski, Aleksei (a cura di)

2011 Readings in Zoosemiotics, Berlino-Boston, De Gruyter Mouton

Marino, Gabriele; Thibault, Mattia (a cura di)

2017 Lexia - Viralità, Torino, Aracne.

Marrone, Gianfranco (a cura di)

2017 Zoosemiotica 2.o, Palermo, Edizioni Museo Pasqualino. 


\section{Dcula ${ }^{21}$}

Geosemiotica: dai locative media, alle immagini diffuse, ai big e small data

Bruno Surace I Visori e galline nel Web of Beings I DOI:10.12977/ocula2019-22

Martinelli, Dario

2010 A Critical Companion to Zoosemiotics. People, Paths, Ideas, Londra-New York, Springer.

Montanari, Federico

2016 "Ancora un "turn"? La svolta Locative nei media e sue possibili implicazioni socio-semiotiche. Casi, esempi, e questioni", in Guido Ferraro e Anna Maria Lorusso, a cura di, Nuove Forme d'interazione: dal web al mobile, Tricase (LE), Libellula, 159-171.

Pocar, Valerio

1998 Gli animali non umani. Per una sociologia dei diritti, Roma-Bari, Laterza.

Putnam, Hilary

1981 Reason, Truth and History, Cambridge, Cambridge University Press.

Surace, Bruno

2017 “Zoosemiotica dei Pokémon”, in Marrone (2017).

Surace, Bruno

2018 "Pokémon and the PETA - Viral Extremeness as a Semiotic Strategy", in Eva Kimminich, Julius Erdmann e Amir Dizdarevi, a cura di, Virality and Morphogenesis of Right Wing Internet Populism, Frankfurt am Main, Peter Lang.

Surace, Bruno

2018 "Baby Simulacra. Semiotica dei cuccioli al cinema come incubatori di assiologie", in Francesco Mangiapane, a cura di, $E \mid C$-Cuccioli, pets e altre carinerie (Rivista on-line dell'AISS - Associazione Italiana Studi Semiotici).

Taddeo, Gabriella

2007 Ipercinema. L’immaginario cinematografico nell'era digitale, Milano, Guerini scientifica.

Thibault, Mattia; Marino, Gabriele

2018 "The True King of the Internet: gattini, gattari e gattese dalle periferie al centro del Web", in Francesco Mangiapane, a cura di, $E \mid C$ - Cuccioli, pets e altre carinerie (Rivista on-line dell'AISS - Associazione Italiana Studi Semiotici).

Bruno Surace è PhD in Semiotica e Media presso l'Università di Torino. Ha all'attivo pubblicazioni peer-reviewed in riviste internazionali. Nel 2017 è stato Visiting Scholar presso lo University College Cork (Eire). Ha tenuto conferenze e seminari in Italia, Europa e Cina. 